\title{
Spontaneous Charging and Crystallization of Water Droplets in Oil
}

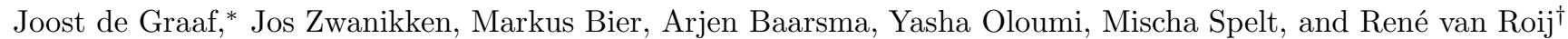 \\ Institute for Theoretical Physics, Utrecht University, \\ Leuvenlaan 4, 3584 CE Utrecht, The Netherlands
}

(Dated: October 24, 2018)

\begin{abstract}
We study the spontaneous charging and the crystallization of spherical micron-sized water-droplets dispersed in oil by numerically solving, within a Poisson-Boltzmann theory in the geometry of a spherical cell, for the density profiles of the cations and anions in the system. We take into account screening, ionic Born self-energy differences between oil and water, and partitioning of ions over the two media. We find that the surface charge density of the droplet as induced by the ion partitioning is significantly affected by the droplet curvature and by the finite density of the droplets. We also find that the salt concentration and the dielectric constant regime in which crystallization of the water droplets is predicted is enhanced substantially compared to results based on the planar oil-water interface, thereby improving quantitative agreement with recent experiments.
\end{abstract}

PACS numbers: 68.05.-n, 64.70.D-, 82.70.Kj

\section{INTRODUCTION}

It is well-established that water and oil do not mix: droplets of water in oil (or droplets of oil in water) tend to coalesce such that the oil-water mixture coarsens until macroscopic phase separation of oil and water is achieved. It is also well-established that this coarsening process can be delayed or even prevented by additives such as surfactants or colloidal particles, which adsorb to the oil-water interface and thereby stabilize the droplets, either thermodynamically (such as in micro-emulsions) or kinetically (such as in Pickering emulsions) [1, 2]. Recently, however, experimental observations by Leunissen and coworkers [3, 4] revealed stable micron-sized water droplets in somewhat polar oils without any additives. In fact, under appropriate conditions the oil-dispersed water droplets could even form a body-centered cubic (bcc) crystalline phase with a lattice spacing of the order of $10 \mu \mathrm{m}$, and some of these crystals have been stable for almost two years now without any observation of droplet coalescence [5]. The mechanism by which these water droplets are stabilized was argued to stem from an asymmetric partitioning of the (ever-present) monovalent cations and anions over the oil and water phase; in the experiments of Refs. [3, 4] with the oil cyclohexyl bromide the ions involved include $\mathrm{H}^{+}$and $\mathrm{Br}^{-}$, and the somewhat larger water affinity of the former compared to the latter should lead to positively charged water droplets. This suggested mechanism was confirmed in theoretical calculations [4, [6] of monovalent ions in the vicinity of a planar oil-water interface, on the basis of Poisson-Boltzmann theory combined with ionic Born self energy in water and oil 7, 8, 9]. Although more advanced models could be invoked, e.g., involving more ionic correlations [10, 11, 12, 13] or a better account of

\footnotetext{
*Electronic address: j.degraaf@phys.uu.nl
}

${ }^{\dagger}$ Electronic address: r.vanroij@uu.nl the ionic self-energy [14, 15, 16, 17], the relatively simple model used in Refs. [4, 6] showed surface charge densities of the order of $10-100$ elementary charges per $\mu \mathrm{m}^{2}$ and hence $100-1000$ charges for micron-sized waterdroplets. With such a droplet charge the observed bcc crystals of water-droplets in oil could be explained, at least qualitatively, and therefore we use this relatively simple Poisson-Boltzmann-Born model for further theoretical explorations.

The theory presented in Refs. [4, 6] considers a planar oil-water interface separating two half spaces of oil and water. The advantage of this assumption lies in the fact that it allows for some analytic expressions for the surface charge density and the ionic contribution to the interfacial tension within nonlinear Poisson-Boltzmann theory [18], which leads to an efficient scheme to analyze the parameter space. However, one could a priori expect quantitative shortcomings due to the assumed planar geometry, e.g., because the typical experimental droplet radius of about $1 \mu \mathrm{m}$ is quite a bit smaller than the typical screening length of about $10 \mu \mathrm{m}$ in the oil phase, or because the typical lattice spacing in the crystal is of the same order as the screening length such that the net charge of the droplets could be affected by the nearby other droplets. In order to investigate these effects we extend in this paper the theory of Refs. [4, [6] from the planar to the spherical geometry. This will be done in the context of a cell model [19], where a single spherical droplet is considered in the center of a spherical cell with a finite volume representing the density of droplets. Whereas this geometry does no longer allow for analytic solutions of the Poisson-Boltzmann equation, the numerical solution is, however, fairly straightforward because of the radial symmetry. This geometry therefore enables us to study simultaneously the effects of droplet-curvature and droplet-density on the ionic double layers and on preferential adsorption and charging in the vicinity of the droplet surface. We will show that these effects, when compared to the planar limit results, give rise to a significantly larger crystallization regime for water-droplets 


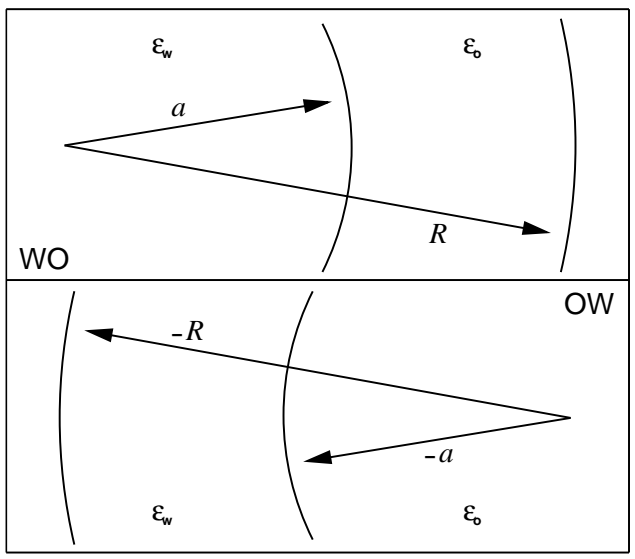

FIG. 1: A single Wigner-Seitz cell with radius $R$ centered on a droplet of radius $a$. To distinguish between a water-in-oil emulsion $(\mathrm{WO},+)$ and an oil-in-water $(\mathrm{OW},-)$ emulsion, a sign convention has been introduced.

in oil (due to a larger surface charge), and to a much smaller surface charge for oil-droplets in water. In fact, our numerical predictions for the crystallization regime are now quantitatively closer to the experimentally observed one, although there is still some deviation that we attribute to other shortcomings and oversimplifications of our microscopic model, e.g., the crude approximation of describing the ionic self-energies in oil and water by a simple Born energy. Our present results indicate, however, that the essential physical mechanism of preferential ion partitioning can indeed explain the crystallization of water-droplets in oil as observed in Refs. [3, 4].

\section{DENSITY FUNCTIONAL THEORY FOR SALINE EMULSIONS}

\section{A. Wigner-Seitz cell approach}

We consider an emulsion of water-in-oil droplets (WO) of total volume $V$ containing $N$ identical droplets with radius $a$. The volume of water in the system is defined as $x V \equiv 4 \pi N a^{3} / 3$, with $x$ the volume fraction, hence $(1-x) V$ is the volume of oil. The theory describing emulsions of oil-droplets in water (OW) is analogous to that for WO systems outlined in this section. The emulsion contains monovalent ions with ionic radii $a_{ \pm}$, which are typically $2-4 \AA$. Using a cell model [19], we reduce the $N$-droplet problem to that of a single droplet in a spherically symmetric Wigner-Seitz cell, see Fig. 1, with radius $R \equiv a x^{-1 / 3}$, such that $V=4 \pi N R^{3} / 3$.

In this single cell we consider a spherical oil-water interface located at $r=a$. The interface separates two bulk phases consisting of water $(0<r<a)$ and oil $(a<r<R)$. Both, oil and water, are considered to be incompressible linear dielectrics, which means that the solvent background is characterized by the relative dielectric constant $\epsilon_{w}$ and $\epsilon_{o}$, respectively, see Fig. 1. The dielectric profile (relative to the dielectric constant of vacuum $\epsilon_{v}$ ) is a step-function $\epsilon(r)=\epsilon_{w}$ if $0<r<a$ and $\epsilon(r)=\epsilon_{o}$ if $a<r<R$. The ions are described by spherically symmetric ionic density profiles $\rho_{ \pm}(r)$. The ions experience Coulombic ion-ion interactions, which we treat in a mean-field fashion, and ion-medium interactions.

The ion-medium interaction is taken into account via an external potential acting on the ions. Due to the dielectric properties of oil and water, the ions have different electrostatic self-energies in the two solvents. Using the Born approximation [9], this self-energy of a cation $(+)$ and an anion (-) is given by $E_{ \pm}\left(\epsilon_{i}\right) \equiv e^{2} /\left(2 \epsilon_{v} \epsilon_{i} a_{ \pm}\right)$, with $e$ the elementary charge and $i=w, o$. This self-energy and the above dielectric profile $\epsilon(r)$, allows us to rewrite the external potential acting on a cation and an anion as $V_{ \pm}(r)=E_{ \pm}(\epsilon(r))-E_{ \pm}\left(\epsilon_{w}\right)$, where we note that this potential is constructed to be zero in water. For realistic $\epsilon_{o} \approx 4-20$ the potential is of order $(1-20) k_{B} T$ in oil, i.e., the ions prefer to be in the water. We also use the notation $V_{ \pm}(r)=0$ if $0<r<a$ and $V_{ \pm}(r)=k_{B} T f_{ \pm}$if $a<r<R$, with $f_{ \pm}$dimensionless and implicitly dependent on $\epsilon_{w}$ and $\epsilon_{o}$. Here $k_{B} T$ is the thermal energy, $k_{B}$ Boltzmann's constant, and $T$ the temperature.

\section{B. Poisson-Boltzmann equation}

Using the above external potential we employ the framework of density functional theory [20, 21] to calculate the equilibrium density profiles $\rho_{ \pm}(r)$. The grandpotential functional $\Omega\left[\rho_{ \pm}\right]$for a single Wigner-Seitz cell can be written as

$$
\begin{aligned}
\beta \Omega\left[\rho_{ \pm}\right]= & 4 \pi \sum_{i= \pm} \int_{0}^{R} r^{2} \rho_{i}(r)\left[\log \left(\frac{\rho_{i}(r)}{\rho_{s}}\right)-1\right. \\
& \left.+\frac{1}{2} q_{i} \phi\left(r,\left[\rho_{ \pm}\right]\right)+\beta V_{i}(r)\right] \mathrm{d} r
\end{aligned}
$$

with $\beta=1 /\left(k_{B} T\right)$ and the ionic valencies $q_{ \pm}= \pm 1$. The first line is the ideal-gas grand-potential functional. The second line describes the ion-ion Coulomb interaction in mean-field approximation and the ion-solvent interactions characterized by the external fields. The chemical potentials are represented in the form of an ion concentration $\rho_{s}$, which is actually the ion concentration in a water reservoir in equilibrium with the emulsion. The electrostatic interactions between the ions in Eq. (11) are given in terms of the electrostatic potential functional $k_{B} T \phi\left(r,\left[\rho_{ \pm}\right]\right) / e$, which satisfies the Poisson equation

$$
\epsilon_{v} \epsilon(r) \nabla^{2} \phi\left(r,\left[\rho_{ \pm}\right]\right)=-4 \pi \beta e^{2} \sum_{i= \pm} q_{i} \rho_{i}(r)
$$

with boundary conditions

$$
\begin{aligned}
\lim _{r \uparrow a} \epsilon_{w} \phi^{\prime}\left(r,\left[\rho_{ \pm}\right]\right) & =\lim _{r \downarrow a} \epsilon_{o} \phi^{\prime}\left(r,\left[\rho_{ \pm}\right]\right) ; \\
\lim _{r \downarrow 0} \phi^{\prime}\left(r,\left[\rho_{ \pm}\right]\right) & =\lim _{r \uparrow R} \phi^{\prime}\left(r,\left[\rho_{ \pm}\right]\right)=0,
\end{aligned}
$$


where the prime denotes a derivative w.r.t. $r$.

Minimizing the grand-potential functional leads to the Euler-Lagrange equation $\delta \Omega / \delta \rho_{ \pm}(r)=0$, which can be rewritten as Boltzmann distributions

$$
\rho_{ \pm}(r)=\rho_{s} \exp \left(-\beta V_{ \pm}(r) \mp \phi\left(r,\left[\rho_{ \pm}\right]\right)\right) .
$$

In practice we implement the condition that $\rho_{ \pm}(0)=\rho_{s}$ for WO emulsions and $\rho_{ \pm}(-R)=\rho_{s}$ for OW emulsions, where we adhere to the sign convention explained in Fig. 1. In the systems studied $\kappa_{w} a \gg 1$, with $\kappa_{w}^{-1}$ the Debye length in water, so that the water phase can indeed be considered a bulk phase and hence acts as a salt reservoir with total ion concentration $2 \rho_{s}$. Using Eq. (5) the Poisson equation reduces to

$$
\nabla^{2} \phi(r)=\kappa(r)^{2} \sinh \left(\phi(r)-\phi_{c}(r)\right) \quad(r \neq a),
$$

where we have introduced $\kappa(r)=\kappa_{w}$ if $0<r<a$ and $\kappa(r)=\kappa_{o}$ if $a<r<R$, with $\kappa_{i}^{2} \equiv 8 \pi \beta e^{2} \rho_{i} /\left(\epsilon_{v} \epsilon_{i}\right)$ in medium $i=o, w$ with $\rho_{w}=\rho_{s}$ and $\rho_{o}=\rho_{s} \exp \left(-\left[f_{+}+\right.\right.$ $\left.f_{-}\right] / 2$ ) the bulk ion concentrations. The Donnan potential $\phi_{c}(r)=\beta\left[V_{-}(r)-V_{+}(r)\right] / 2$ follows from the local charge neutrality in the bulk liquids. Note that we have dropped the explicit $\left[\rho_{ \pm}\right]$dependence from $\phi\left(r,\left[\rho_{ \pm}\right]\right)$in Eq. (6), since the above differential equation is not explicitly $\rho_{ \pm}$dependent. Equation (6) together with its boundary conditions (see Eqs. (3) and (44)) has a unique solution and can be solved numerically on an $r$-grid by employing standard numerical algorithms. Typically we require several thousand non-equidistant grid points, with a relatively small grid spacing close to $r=a$, tailored to the screening length in the oil and water phase, respectively.

\section{Ion-induced physical quantities}

Using the numerical solution for $\phi(r)$ (and hence for $\rho_{ \pm}(r)$ ) we can determine the charge and the excess interfacial tension of the droplet, and the inter-droplet coupling parameter. The number of net unit charges induced by ion partitioning on the droplet is given by

$$
Z=4 \pi \sum_{i= \pm} \int_{0}^{a} r^{2} q_{i} \rho_{i}(r) \mathrm{d} r .
$$

Two charged water droplets, at separation $r>2 a$, are assumed to interact with each other through a screened Coulomb droplet-droplet interaction potential

$$
U(r)=\frac{Z^{2} e^{2}}{\epsilon_{v} \epsilon_{o}} \frac{\exp \left(\kappa_{o}(2 a-r)\right)}{\left(1+\kappa_{o} a\right)^{2} r} .
$$

We introduce the coupling parameter

$$
\Gamma \equiv \beta U\left(\rho^{-1 / 3}\right)\left(1+k+k^{2} / 2\right),
$$

with $k=\kappa_{o} \rho^{-1 / 3}$ and $\rho^{-1 / 3}=(4 \pi / 3)^{1 / 3} R$, i.e., $\rho=N / V$ the droplet density. It is empirically known from pointYukawa $\left(\kappa_{o} a=0\right)$ simulations 22] that crystallization occurs when $\Gamma>106$. Even though the systems of present interest do have a finite 'hard' core, we can still apply this freezing criterion, because $U(2 a) \gg k_{B} T$ and $\kappa_{o} a \lesssim 1$ for most of our parameters, i.e., the repulsions are dominated by the screened-Coulomb part rather than the hard core.

The excess interfacial tension $\gamma$ induced by ionic partitioning is defined as $\gamma \equiv\left(\Omega\left[\rho_{ \pm}\right]-\Omega\left[\rho_{h}\right]\right) /\left(4 \pi a^{2}\right)$, where $\rho_{h}(r)=\rho_{w}$ if $0<r<a$ and $\rho_{h}(r)=\rho_{o}$ if $a<r<R$, i.e., the difference between the grand-potential of the system and the grand-potential of a homogeneous reference system (per area). Using the above definition, $\gamma$ can be rewritten as

$$
\beta \gamma=-\frac{1}{a^{2}} \sum_{i= \pm} \int_{0}^{R} r^{2}\left[\rho_{i}(r)-\rho_{h}(r)+\frac{1}{2} q_{i} \rho_{i}(r) \phi(r)\right] \mathrm{d} r .
$$

The term 'excess' refers to the fact that the total interfacial tension reads $\gamma_{\text {tot }}=\gamma_{\text {bare }}+\gamma$, where $\gamma_{\text {bare }}$ is the bare oil-water interfacial tension, i.e., for an oil-water interface without the presence of ions. This bare interfacial tension is positive and typically of the order $1-10$ $\mathrm{mN} / \mathrm{m}$, whereas $\gamma$ turns out to be negative and of the order $10-100 \mathrm{nN} / \mathrm{m}$.

\section{RESULTS AND DISCUSSION}

\section{A. Choice of parameters}

From the experiments of Refs. [3, 4] we know that crystallization of water-in-oil droplets has been observed for the following parameters. Water has $\epsilon_{w}=80.0$ at $T=293 \mathrm{~K}$. For water-droplets in CHB (cyclohexyl bromide) we find from an analysis of the published snapshots that $a=1.1 \pm 0.2 \mu \mathrm{m}$ and $l=9.6 \pm 0.8 \mu \mathrm{m}$, with $l$ the nearest neighbor distance in a bcc crystal [3]. Elementary geometry shows that the volume fraction of water is then given by $x=\pi \sqrt{3}(a / l)^{3}$, which yields $x=(8.1 \pm 1.6) \cdot 10^{-3}$. For CHB $\epsilon_{o}=7.9$ and electroconductivity measurements give an indication of the bulk salt concentrations $\rho_{w}$ and $\rho_{o}$ [3]. According to Ref. [4] the major constituent ions are $\mathrm{H}^{+}, \mathrm{OH}^{-}$and $\mathrm{Br}^{-}$, with $a_{\mathrm{H}^{+}}=2.8 \AA$ and $a_{\mathrm{Br}^{-}}=3.3 \AA[3]$. Likewise, for waterdroplets in a CHB-decalin mixture (see Ref. 3] for details) we find $a=1.3 \pm 0.2 \mu \mathrm{m}, l=16 \pm 2 \mu \mathrm{m}$ and hence $x=(2.9 \pm 0.6) \cdot 10^{-3}$. This CHB-decalin mixture has $\epsilon_{o}=5.6$ and $\kappa_{o}^{-1} \gtrsim 3.6 \mu \mathrm{m}$ [4]. Again the contributing ions are $\mathrm{H}^{+}, \mathrm{OH}^{-}$and $\mathrm{Br}^{-}$, but their respective concentrations in CHB-decalin or water have a high degree of uncertainty.

In our theoretical investigation we have chosen system parameters in the range indicated by the experiments of Ref. [3]. However, to capture the physics of the curvature effects present at spherical interfaces we do not fully take into account the complex chemistry described above. Our basis parameter set is $R=10 \mu \mathrm{m}, a=1 \mu \mathrm{m}, \rho_{w}=10^{-3}$ $\mathrm{M}, \epsilon_{w}=80, \epsilon_{o}=5, a_{+}=3.6 \AA$ and $a_{-}=3.0 \AA$. We vary 


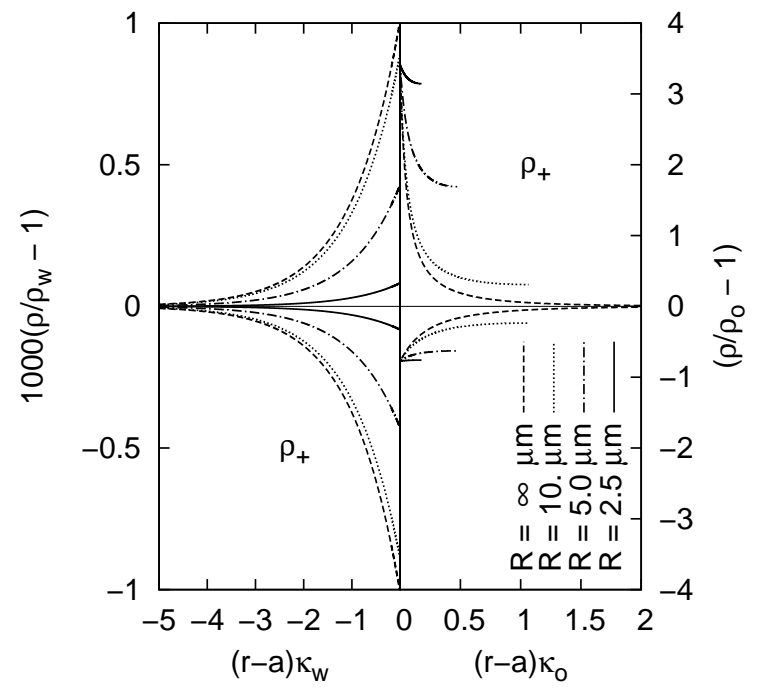

FIG. 2: The double-layer near the interface of a spherical water-in-oil droplet, showing ion partitioning of the ions. Here $a=1 \mu \mathrm{m}, \rho_{w}=10^{-3} \mathrm{M}, \epsilon_{w}=80, \epsilon_{o}=5, a_{+}=3.6 \AA$ and $a_{-}=3.0 \AA$, which gives $\kappa_{w}^{-1}=9.63 \mathrm{~nm}, \kappa_{o}^{-1}=8.44 \mu \mathrm{m}$ and $\rho_{o}=8.15 \cdot 10^{-11} \mathrm{M}$. The deviation from the homogeneous density profile is given as a function of the distance from the interface measured in screening lengths, for several values of the Wigner-Seitz cell radius $R$. The upper-left and lower-right quadrants correspond to $\rho_{-}(r)$ profiles, whereas the lower-left and upper-right correspond to the $\rho_{+}(r)$ profiles. Note that the lines on the oil side of the interface terminate at $(R-a) \kappa_{o}$, i.e., $r=R$.

one or more of these parameters at a time and examine the effect on the physical quantities $Z, \gamma$, and $\Gamma$. For this basis parameter set we have $f_{+}=14.8, f_{-}=17.8$ (using the Born approximation), $x=10^{-3}, \rho_{o}=8.15 \cdot 10^{-11}$ $\mathrm{M}, \kappa_{w}^{-1}=9.63 \mathrm{~nm}$ and $\kappa_{o}^{-1}=8.44 \mu \mathrm{m}$. We take two ionic species for simplicity and numerical convenience. The positive ionic radius corresponds to that of $\mathrm{Na}^{+}$and the negative to that of $\mathrm{Cl}^{-}[23,24,25]$. At this point the choice for the ion concentration in water seems a bit arbitrary, but we will show that it is in fact reasonable.

The results for spherical interfaces have been calculated using numerical techniques, whereas in the planar limit results can be determined analytically [18]. Note that the planar limit describes flat oil-water interfaces, therefore the planar system should correspond to a spherical Wigner-Seitz cell with $a, R-a \gg \kappa_{o}^{-1}$, i.e., the interface is locally flat on the scale of the Debye length of oil; we will denote this limit as $a, R \rightarrow \infty$.

\section{B. Preliminary analysis}

Figure. 2 shows the ionic density profiles for an $a=1$ $\mu \mathrm{m}$ water-in-oil droplet near the interface for several Wigner-Seitz cell radii $R$. Note the different scaling of all four axes, and that $x$ increases from $0(R \rightarrow \infty)$,
TABLE I: Physical quantities corresponding to Fig. 2 compared to those calculated analytically for a planar system.

\begin{tabular}{cccccc}
\hline \hline $\begin{array}{c}a \\
(\mu \mathrm{m})\end{array}$ & $\begin{array}{c}R \\
(\mu \mathrm{m})\end{array}$ & $\begin{array}{c}\sigma^{a} \\
\left(\mathrm{e} / \mu \mathrm{m}^{2}\right)\end{array}$ & $\begin{array}{c}Z \\
(\mathrm{e})\end{array}$ & $\begin{array}{c}\gamma \\
\mathrm{nN} / \mathrm{m}\end{array}$ & $\begin{array}{c}\Gamma \\
-\end{array}$ \\
\hline$\infty$ & $\infty^{b}$ & -1.34 & $-\infty$ & -3.7 & - \\
1.0 & $\infty$ & -11.61 & -145.9 & -34.8 & 0.0 \\
1.0 & 10.0 & -10.23 & -128.5 & -30.6 & 16.8 \\
1.0 & 5.0 & -4.93 & -61.9 & -14.0 & 8.7 \\
1.0 & 2.5 & -0.94 & -11.8 & -2.4 & 0.64 \\
\hline \hline
\end{tabular}

${ }^{a} \sigma \equiv Z /\left(4 \pi a^{2}\right)$

${ }^{b}$ A planar system, where only $\sigma$ and $\gamma$ can be determined.

to $0.001(R=10 \mu \mathrm{m})$, to $0.008(R=5 \mu \mathrm{m})$, to 0.064 $(R=2.5 \mu \mathrm{m})$. Ion partitioning causes the water phase to become negatively charged for $a_{+}>a_{-}$, whereas the oil phase picks up an equal but opposite charge. Note that local charge neutrality at $r=R$ is violated for the finite Wigner-Seitz cells. This finite cell 'compresses' the double-layer inside the droplet w.r.t. that of the planar limit system, resulting in a droplet charge reduction, as can be seen from the shrinkage of the area enclosed by $\rho_{+}(r)$ and $\rho_{-}(r)$ at the water side in Fig. 2. Of course, the same area-shrinkage occurs in the oil phase by the condition of global charge neutrality, however, this effect is not clearly visible. The value of the physical quantities for the various systems in Fig. 2 is shown in Table [ together with the values for the corresponding planar system. Note that we have introduced $\sigma \equiv Z /\left(4 \pi a^{2}\right)$, which is henceforth referred to as the 'surface charge density' of the droplet. The total charge of a planar 'droplet' is infinite, because of its infinite surface area. The results in Table \show that the physical quantities $Z, \gamma$, and $\Gamma$ are highly sensitive to the size $R$ of the Wigner-Seitz cell in the experimentally relevant regime $R \approx 5-10 \mu \mathrm{m}$, $\epsilon_{o} \approx 5$, emphasizing the importance of curvature. We will examine this dependence more closely for extremely dilute emulsion, i.e., those emulsions which can be modelled by a droplet in an infinitely large Wigner-Seitz cell.

\section{EXTREMELY DILUTE EMULSIONS}

\section{A. Droplet size and curvature effects}

We now disentangle the effects of a finite droplet density (finite $R$ ) and that of droplet curvature (finite) $a$ by studying the curvature dependence in the extremely dilute limit $(R \rightarrow \infty)$. In Fig. 3 the droplet's surface charge density $\sigma$ and the excess interfacial tension $\gamma$ as a function of $1 / a$ are shown in the extremely dilute limit. For the systems considered in Fig. 3, it follows that there is correspondence between the analytic planar results and the limit $a \rightarrow \infty$, extrapolated from the spherical results, within a fractional uncertainty of $\approx 10^{-4}$. Data was obtained for sufficiently large $a$ to safely extend the lines 
through the point $1 / a=0$. The surface charge density of the systems considered here $(a>100 \mathrm{~nm})$ lies in the $1-100 e / \mu \mathrm{m}^{2}$ range. We thus find that $a=1 \mu \mathrm{m}$ droplets have charges between $10-1000 e$. The excess interfacial tension ranges between $10-100 \mathrm{nN} / \mathrm{m}$ and is negative. The sign for this interfacial tension is a consequence of the external potential model we use here. We refer to Ref. [26] for a more in-depth discussion of the excess interfacial tension of an interface separating two electrolyte solutions and the way in which it can be modelled.

The curves in Fig. 3 show that there are two asymmetries between OW and WO emulsions. Firstly, the deviation from the planar limit value is linear in $1 / a$ in the case of WO systems $(a>0)$ and non-linear for OW systems $(a<0)$. This asymmetry can be explained entirely by the fact that $\kappa_{w} a \gg 1$ for the WO emulsions considered, whereas $\kappa_{o} a \ll 1$ for the OW emulsions. The deviation is linear for OW systems only in the small regime near $1 / a=0$ where $\kappa_{o} a>1$. For $\kappa_{w} a \approx 1(1 / a \approx 100$ $1 / \mu \mathrm{m})$ the deviation becomes non-linear in WO emulsions as well, however, this is well beyond the scale used in Fig. 3. Secondly, with decreasing droplet radius, $|\sigma|$ and $|\gamma|$ decrease for OW systems, but these quantities increase for WO emulsions. This effect can be attributed to the double-layer modification which occurs in spherical systems. It can be shown that the oil phase imposes the total structure of the double-layer and hence determines the surface charge density and excess interfacial tension. Curvature compresses the oil part of the double-layer in OW emulsions, resulting in a reduction of $|\sigma|$ and $|\gamma|$, whereas the oil part of the WO double-layer gets stretched resulting in a corresponding increase of these quantities. Note that the regime in which the planar limit approximation gives accurate results for $\sigma$ and $\gamma$, i.e., a deviation of less than say $20 \%$, is quite small. For an OW emulsion with $\epsilon_{o} \lesssim 5$ the droplet's surface charge density is negligible for experimentally reasonable droplet radii [3]. The planar value is an upper bound for the quantities $\sigma$ and $\gamma$ in extremely dilute OW emulsion. In WO emulsions, there may be an increase by $50 \%$ (or sometimes much more, see Table (I) for $a=1 \mu \mathrm{m}$ due to curvature effects.

\section{B. Curvature expansions}

It is known for fluids with all intrinsic (correlation) length scales smaller than all geometrical length scales that the deviation of any intensive quantity from the planar value is a linear combination of mean and Gaussian curvature only [27]. In the spherical geometry considered here this statement translates into a surface charge density as a function of the droplet radius $a$ of the form

$$
\sigma(a)=\sigma_{p}\left(\operatorname{sign}(a)+\frac{c_{1}}{\left|\kappa_{o} a\right|}-\frac{c_{2}}{\left|\kappa_{o} a\right|^{2}}\right)
$$

with $c_{1}$ and $c_{2}$ coefficients and $\sigma_{p}$ the analytically known planar value for WO emulsions, provided $\kappa_{w}^{-1}, \kappa_{o}^{-1} \ll$
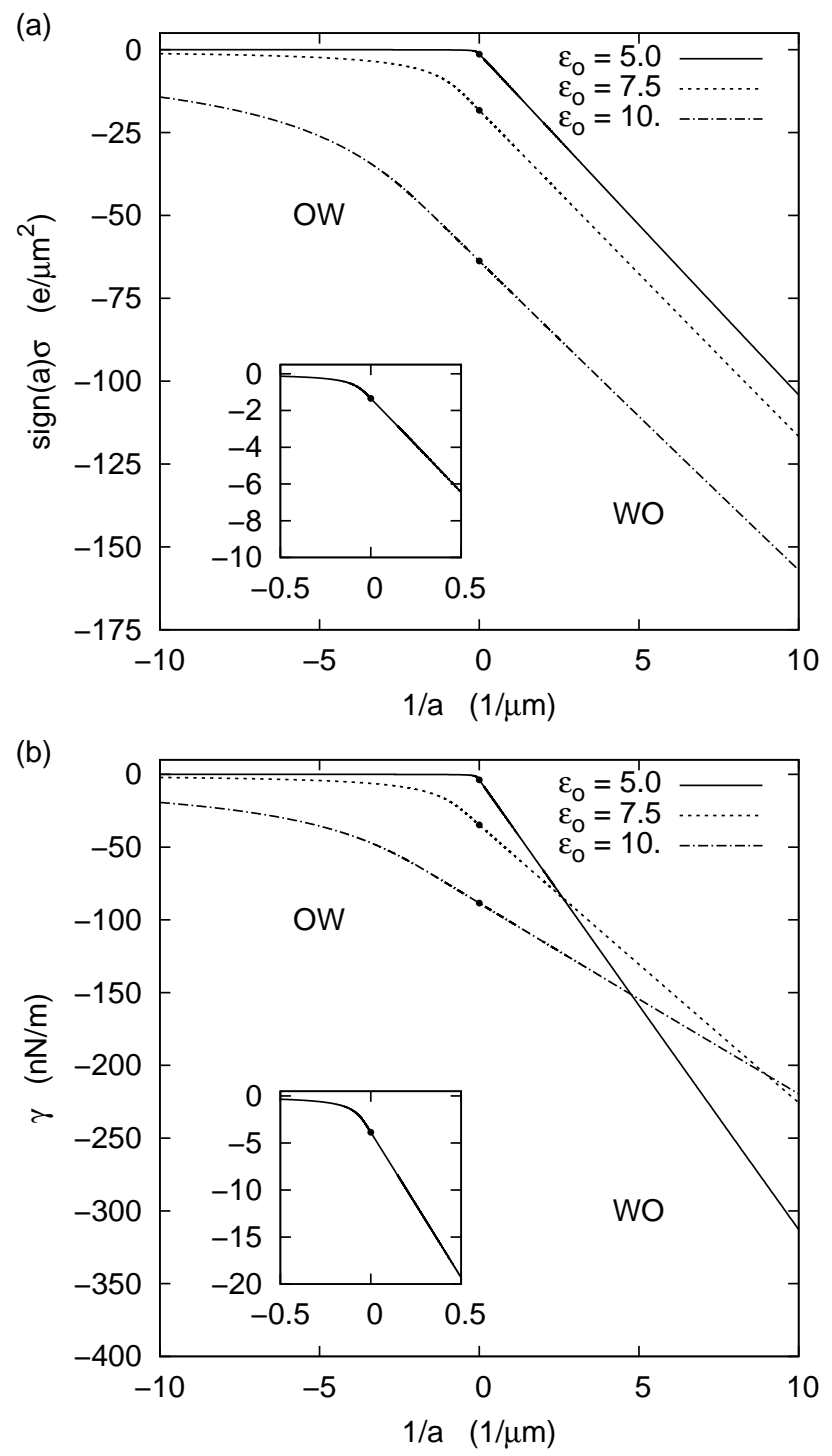

FIG. 3: The surface charge density $\sigma$ (a) and the excess interfacial tension $\gamma(\mathrm{b})$ for an extremely dilute system $(R \rightarrow \infty)$ with salt concentration in water $\rho_{w}=10^{-3} \mathrm{M}$, as a function of the water-droplet radius $a$ at several relative dielectric constants $\epsilon_{o}$ of oil. As specified in Fig. 1 positive values of $a$ correspond to WO systems and negative values to OW systems. The inset shows a enlargement of the area close to the origin. The analytic planar limit values are indicated with dots. Note that the water-in-oil droplets have negative charge, whereas the oil-in-water droplets have positive charge.

$a, R$. We use the sign convention introduced in Fig. 1, which ensures that Eq. (11) is valid for both OW and WO systems. A similar expression can be found for $\gamma$, by replacing $\sigma_{p}$ with $\operatorname{sign}(a) \gamma_{p}$. Moreover, $c_{1}$ and $c_{2}$ are positive and for typical system parameters of order unity. Equation (11) proves useful as it allows us to describe the behavior of emulsion droplets for a range of droplet radii by determining the charge and excess interfacial tension for only two values of the droplet radius, fixing $c_{1}$ and $c_{2}$ in combination with the planar value. If the intrinsic and 

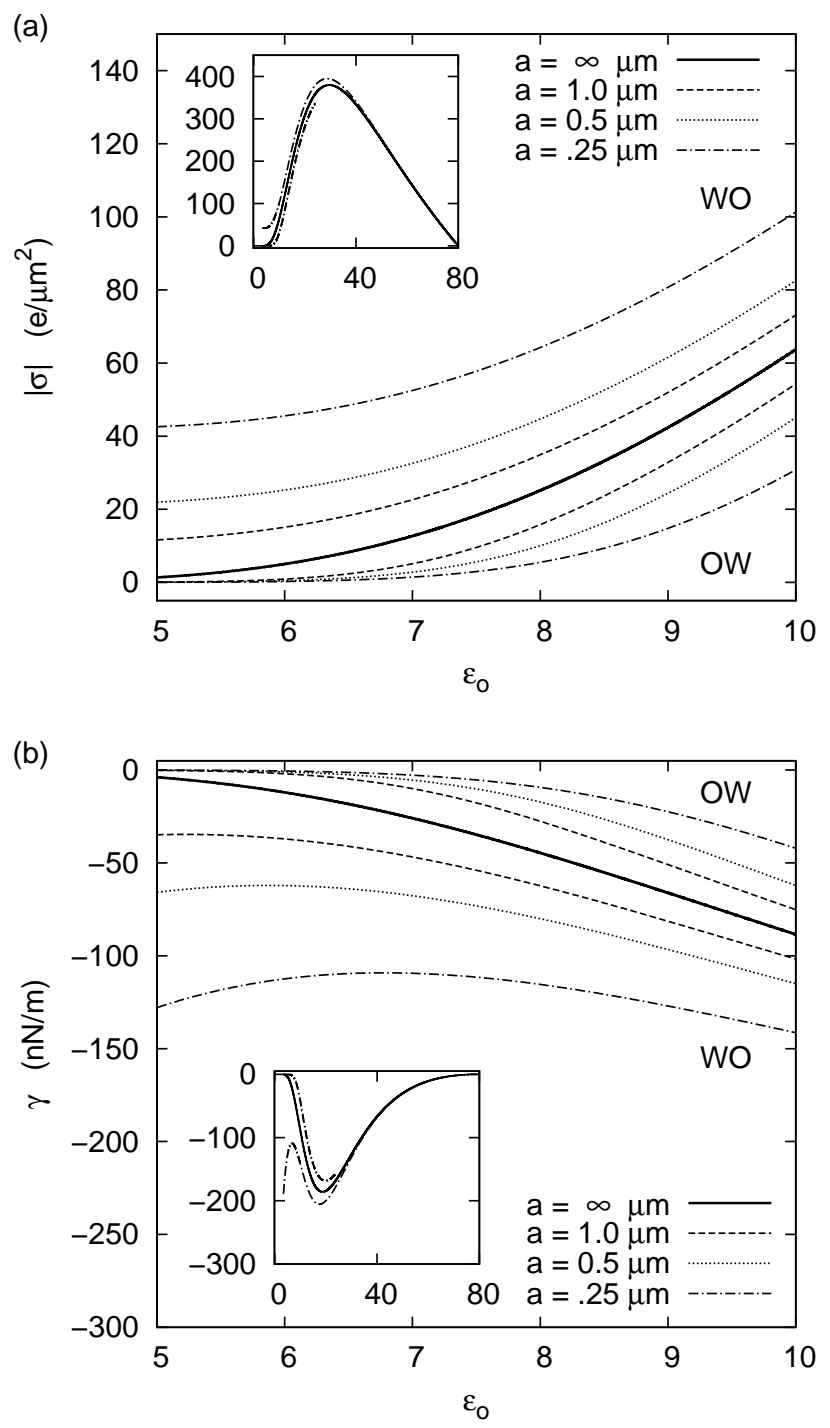

FIG. 4: The surface charge density $\sigma$ (a) and the excess interfacial tension $\gamma$ (b) as a function of the relative dielectric constant of oil $\epsilon_{o}$ for an extremely dilute emulsion, with $\rho_{w}=10^{-3}$ M. Several droplet radii, $a=0.25,0.5$, and 1.0 $\mu \mathrm{m}$, are compared with the analytic planar values $(a \rightarrow \infty)$. The charge of the droplets in OW emulsions is positive and in WO emulsions negative. The insets show the behavior of $\sigma$ and $\gamma$ for the larger domain $3<\epsilon_{o}<80$, with both the planar and the $a=0.25 \mu \mathrm{m}$ result (OW and WO) indicated. The OW line terminates due to numerical instabilities.

the geometric length scales are not well separated, higher order terms in mean and Gaussian curvature can appear in Eq. (11) [27]. However, these higher order terms turn out to be small in this study, since Eq. (11) quantitatively accounts for our numerical data in the droplet radius regime $|a|>0.1 \mu \mathrm{m}$ even though $\kappa_{o}^{-1} \gtrsim a$ in part of this regime.

\section{Varying $\epsilon_{o}, \rho_{w}$ and the droplet radius}

The effect of double-layer modification on a droplet in an extremely dilute system can also be evaluated when we vary $\epsilon_{o}$ and $\rho_{w}$ in an experimentally reasonable range. In Fig. 4, $\sigma$ and $\gamma$ are given as a function of $\epsilon_{o}$, with $R \rightarrow \infty$ and $\rho_{w}=10^{-3} \mathrm{M}$, for several droplet radii $a$. From the insets, which show the full $\epsilon_{o}$-regime, we can see that the planar limit approximation is very accurate in a large $\epsilon_{o}$-range. However, it becomes apparent that in the experimentally relevant $\epsilon_{o}$-range there is a significant deviation from the planar value. In fact, for $\epsilon_{o}<7$ we see that OW systems hardly experience any electrostatic effects $(\sigma \approx 0, \gamma \approx 0)$, whereas for WO emulsions such effects are much stronger than planar theory predicts. We find that $\sigma$ is of the order $1-100 e / \mu \mathrm{m}^{2}$ and $\gamma$ is of order $10-100 \mathrm{nN} / \mathrm{m}$ and negative.

Again there is an asymmetry between OW and WO systems, which can be explained by the difference in Debye length w.r.t. the droplet size. Note that the asymmetry between OW and WO becomes smaller when the dielectric constant of the oil increases and hence the Debye length in oil decreases. Between the two limiting cases $\epsilon_{o}=1$ and $\epsilon_{o}=\epsilon_{w}$, for which both $\sigma$ and $\gamma$ are negligible in the former case and must vanish in the latter case (in our model), we find an extremal value for $\sigma$. This extremum is explained by an increase in $\rho_{o}$, but a decrease in $f_{ \pm}$for $\epsilon_{o} \rightarrow \epsilon_{w}$, and vice versa for $\epsilon_{o} \rightarrow 1$. Note, however, that $\gamma$ seems to diverge for the smallest water-in-oil droplet that we consider here $(a=0.25 \mu \mathrm{m})$ in the limit of small $\epsilon_{o}$ shown in the inset of Fig. 4b. A similar, non-vanishing behavior can be observed for $\sigma$ in the inset of Fig. 怗, however, we do not find an apparent divergence in $\sigma$ for the $\epsilon_{o}$-values considered here. This is in contradiction with the intuitive idea that our model should have negligible surface charge and excess surface tension in the $\epsilon_{o} \rightarrow 1$ limit. Our theoretical investigation cannot exclude instabilities and uncertainties in the numerical algorithm used to solve for $\phi(r)$ in the extreme $\epsilon_{o}$-regime. A more detailed evaluation of the limiting behavior for WO and OW emulsions with $\epsilon_{0} \approx 1$ (e.g., water-droplets in air and air-bubbles in water respectively) with a spherical interface is beyond the scope of this work.

In Fig. 5 we show $\sigma$ and $\gamma$ as a function of $\rho_{w}$ in an extremely dilute system with $\epsilon_{o}=7.5$ for several droplet radii $a$. One can see that the behavior of the physical quantities for spherical interfaces w.r.t. their planar counterparts is analogous to that found in Fig. 4. This analogy can be easily explained by the way in which the Debye lengths are modified when changing either $\epsilon_{o}$ or $\rho_{w}$. Note the values of $\sigma$ and $\gamma$ are in the range $1-100$ $e / \mu \mathrm{m}^{2}$ and $10-100 \mathrm{nN} / \mathrm{m}$, respectively. Our results for extremely dilute emulsions, Figs. 3, 4, and 5, thus show that for reasonable choices of the system's parameters $|\gamma|$ is of the order $10-100 \mathrm{nN} / \mathrm{m}$. This excess interfacial tension therefore does not contribute significantly to the bare interfacial tension of an oil-water interface, which is 

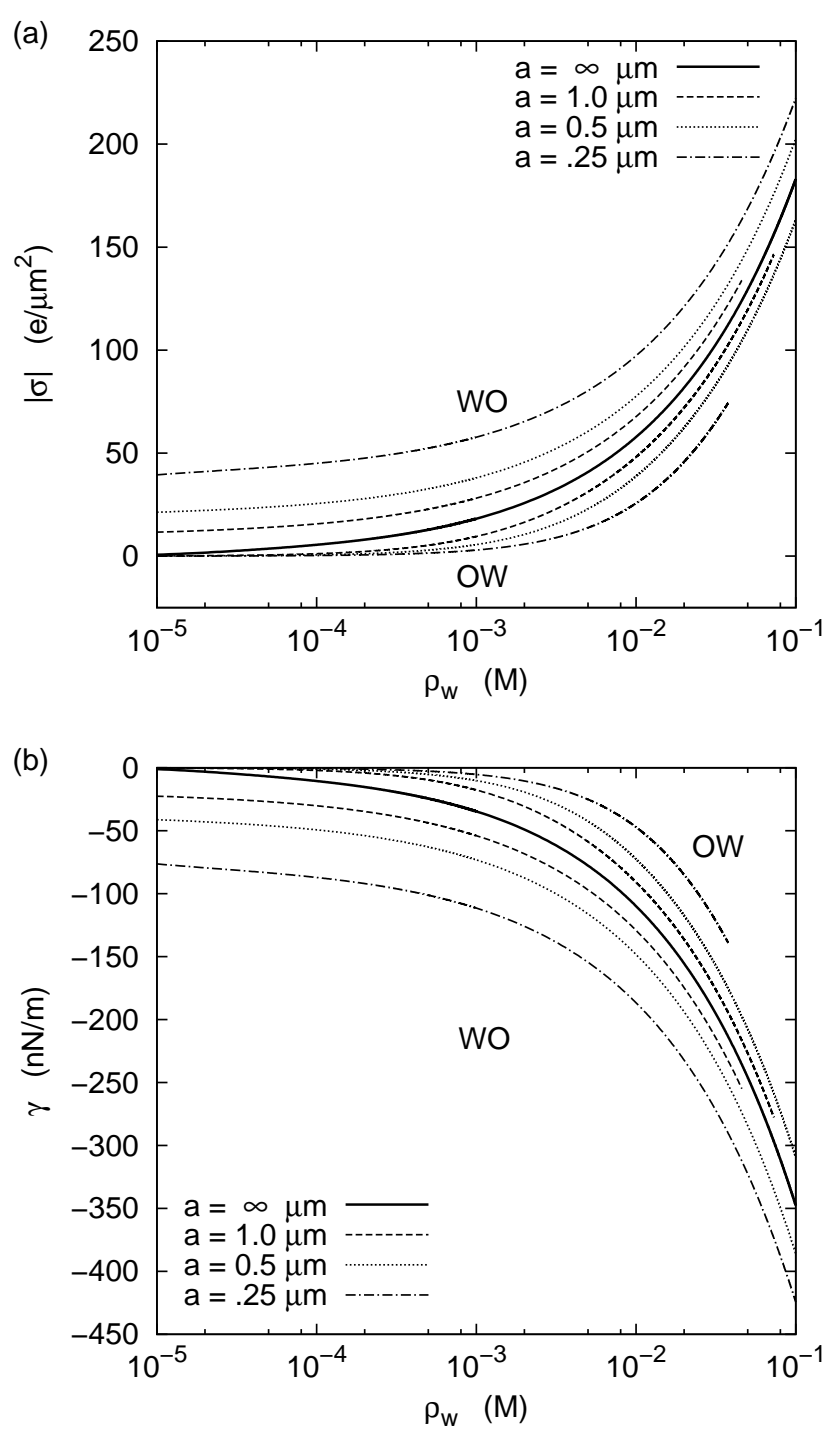

FIG. 5: The surface charge density $\sigma$ (a) and the excess interfacial tension $\gamma$ (b) as a function of the bulk ion concentration in water $\rho_{w}$ for an extremely dilute emulsion with $\epsilon_{o}=7.5$. Several droplet radii, $a=0.25,0.5$, and $1.0 \mu \mathrm{m}$, are considered and can be compared to the planar value. The charge of the droplets in OW emulsions is positive and in WO emulsions negative. Some lines terminate due to numerical instabilities.

of the order $1-10 \mathrm{mN} / \mathrm{m}$.

\section{CRYSTALLIZATION AT FINITE DROPLET VOLUME FRACTION}

In this section we consider a Wigner-Seitz cell with $R=10 \mu \mathrm{m}$, yielding a bcc nearest neighbor distance $l=(\sqrt{3} \pi)^{1 / 3} R=17.6 \mu \mathrm{m}$, which for $\epsilon_{o} \approx 5$ is within the regime for the experiments of Refs. [3, 4]. Only WO emulsions are examined, as we found no crystallization for OW emulsions, i.e., $\Gamma \ll 106$ (see Eq. (9)) for any reasonable choices of the OW system parameters. Our model thus predicts that crystallization of oil-in-water droplets does not occur or is extremely unlikely. Figure 6 indicates for WO systems the $\Gamma=106$ isoline, which is in fact the WO droplet freezing line, in the $\left(\epsilon_{o}, x\right)$-plane (a) and the $\left(\rho_{w}, x\right)$-plane (b) for our basis parameter set. The choice for the $\epsilon_{o}$-range in Fig. 6a is inspired by the range of dielectric constants for which droplet crystal formation has been observed [3]. The $\rho_{w}$-range in Fig. 6 $\mathrm{b}$ is physically reasonable, inspired by the isoline minimum found in planar analysis, and limited by the stability of our numerical algorithm to solve for $\phi(r)$ in the spherical geometry.

We see that there is an isoline minimum at $\epsilon_{0} \approx 5.5$ $\left(\rho_{w}=10^{-3} \mathrm{M}\right)$ and at $\rho_{w} \approx 3.0 \cdot 10^{-3} \mathrm{M}\left(\epsilon_{0}=5\right)$ with $x \approx 0.025$ for the spherical results. This value is substantially smaller than that of the planar minimum $(x \approx 0.065)$, however it is still significantly larger than the experimentally found water content of emulsions in which water-in-oil crystals were observed. This was to be expected if one considers the uncertainty of some of the parameters used, particularly the ionic contents of the emulsions and the corresponding ionic self-energies. The location of the minimum also gives an a posteriori justification of our choice to use $\rho_{w}=10^{-3} \mathrm{M}$ for our basis parameter set. Note the regime in which crystallization can occur according to spherical theory is greatly extended with respect to that found using planar theory. Yet there are parameter choices for which this regime is reduced, also see Fig. 6. Therefore, we must conclude that the effects of curvature on the crystallization of spherical water-in-oil droplets are non-trivial and involve competing processes: at extreme dilution the surface charge density is higher than the planar value, but at finite concentration it becomes smaller, also see Table I. In accordance with the rough location of the minimum found in Fig. [6] we will use $\epsilon_{o}=5$ and $\rho_{w}=10^{-3} \mathrm{M}$ to examine the effects of the self-energy difference between water and oil of the respective ions, keeping $R=10 \mu \mathrm{m}$ $(l=17.6 \mu \mathrm{m})$ fixed.

In Fig. $7 \mathrm{~b}$ the $\Gamma=106$ isolines are indicated as a function of $f_{ \pm}$for several $\rho_{w}$ and $x=10^{-3}$, together with the convex envelope of the isolines within the $\rho_{w}=10^{-7}-10$ $\mathrm{M}$ range for both spherical and planar interfaces. The choice of this $\rho_{w}$ envelope range is inspired by well-known numbers for the ion concentration in water, which is bounded from below by that of pure water with a $\mathrm{pH}$ of 7 caused by self-dissociation of water molecules and from above by that of a saturated solution of order $10 \mathrm{M}$. Note that crystallization of water-droplets in oil can occur in the region enclosed by the isolines or envelopes, respectively, and the $f_{+}$-axis, i.e., in this region $\Gamma>106$. The spherical interface envelope in Fig. $7 \mathrm{l}$ was determined by calculating the $\Gamma=106$ isolines for $\rho_{w}=10^{-6}-10^{-2} \mathrm{M}$. All of these isolines turn out to be shifted in the same way with respect to their planar counterparts as is shown explicitly for $\rho_{w}=10^{-3} \mathrm{M}$. Hence, we have assumed that this behavior can be extrapolated to $\rho_{w}=10 \mathrm{M}$. Note 
(a)

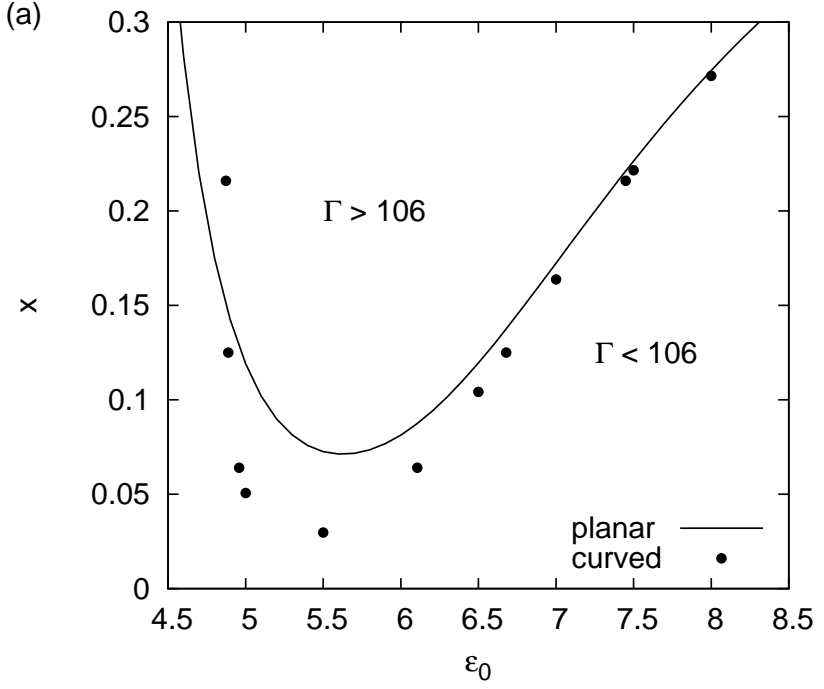

(b)

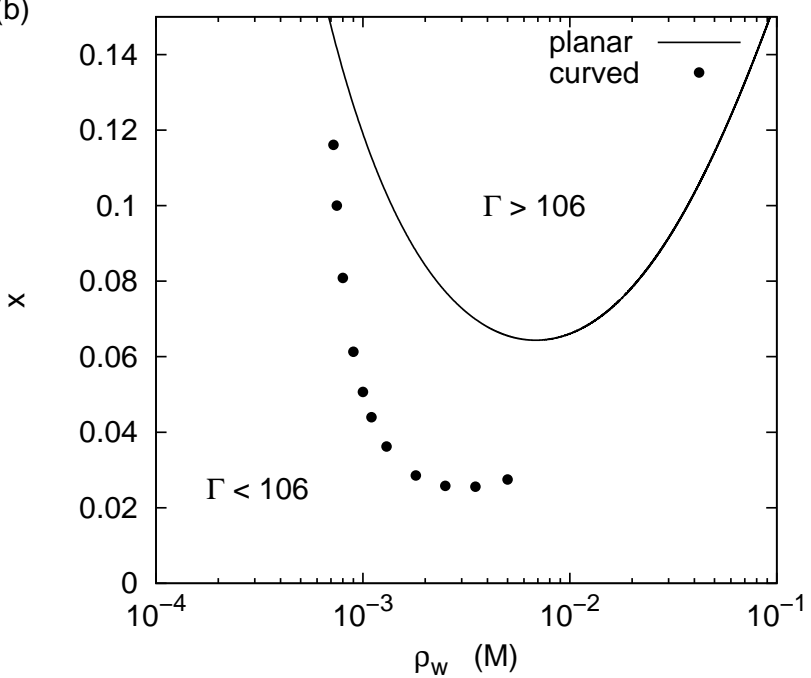

FIG. 6: The freezing line, $\Gamma=106$, as a function of the relative dielectric constant of oil $\epsilon_{o}$ and the water volume fraction $x$ for $R=10 \mu \mathrm{m}$ and $\rho_{w}=10^{-3} \mathrm{M}$ (a) and as a function of $\rho_{w}$ and $x$ for $R=10 \mu \mathrm{m}$ and $\epsilon_{o}=5(\mathrm{~b})$. The planar $\Gamma=106$ isoline is also indicated and several data points which lie on the spherical interface freezing line are included. Above the isolines $\Gamma>106$ and below $\Gamma<106$. Note the shift in the freezing line minima and the increase in the crystallization zones.

again that the effect of curvature is to increase the range over which crystallization can occur.

Fig. $7 \mathrm{~b}$ shows these convex envelopes, in the planar limit approximation, as a function of $f_{ \pm}$for several $x$ and $\epsilon_{o}$. In agreement with our findings in Fig. [6] we recover that crystallization occurs more easily for larger $x$, as one would expect, since larger droplets have a higher charge and are closer together when $R$ is fixed. Note that in the $2.5-10 \epsilon_{o}$-range there is no significant shift in the convex envelopes and their corresponding $\Gamma=106$ isolines. The spherical interface results have not been considered
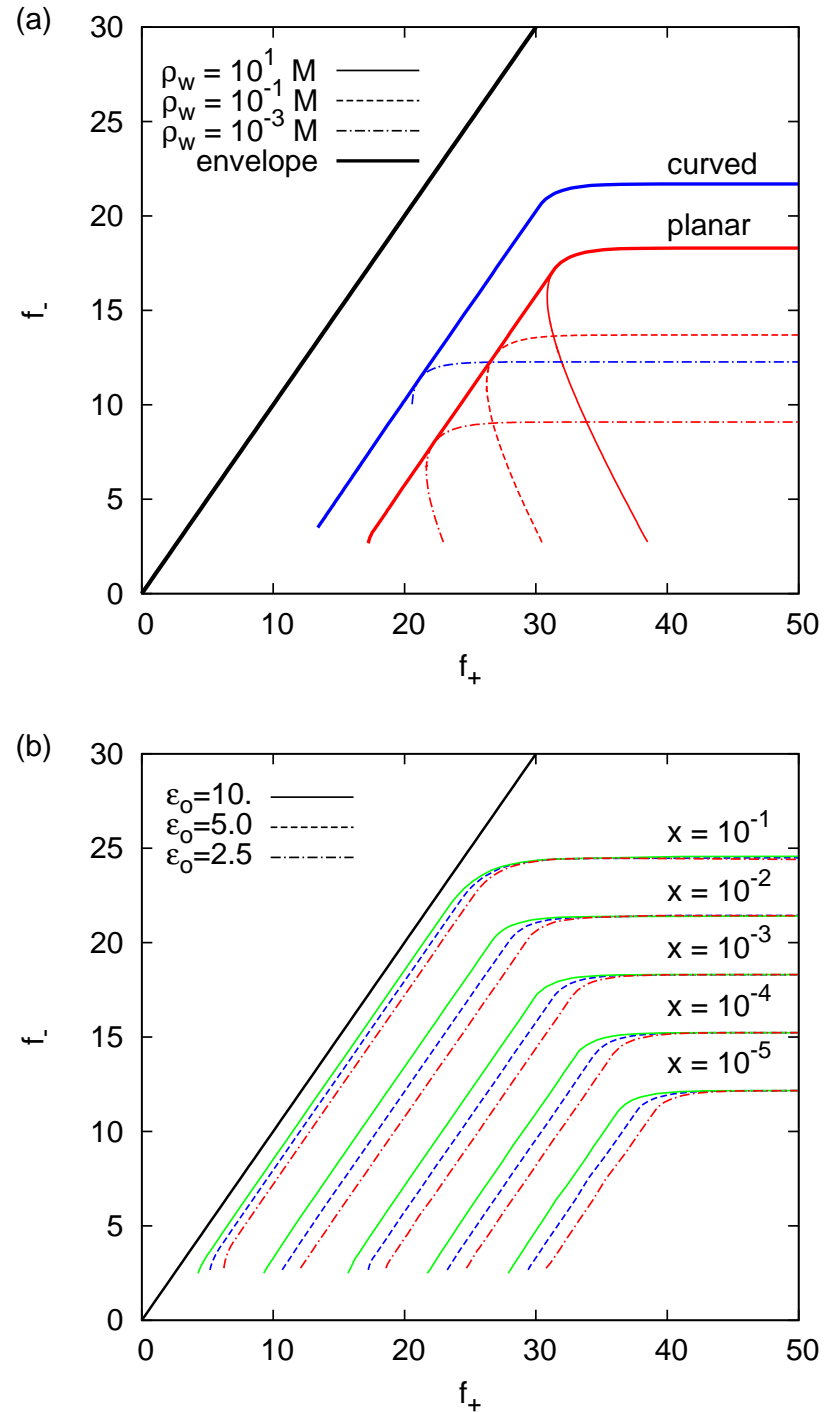

FIG. 7: (Color online) Coupling parameter isolines $\Gamma=106$ for water-in-oil droplets in the $\left(f_{+}, f_{-}\right)$plane (see text), in (a) for several salt concentrations $\rho_{w}$ (dashed) and the $\rho_{w^{-}}$ envelopes (full curves) based on both curved and planar geometries at composition $x=10^{-3}$ and oil dielectric constant $\epsilon_{o}=5$, in (b) only the $\rho_{w}$-envelopes at several $x$ and $\epsilon_{o}$ based on the planar geometry. In all cases the droplet density is kept fixed such that $R=10 \mu \mathrm{m}$. The diagonal $\left(f_{+}=f_{-}\right)$ is a reflection symmetry axis for all curves. Crystallization is predicted between the $f_{+}$axis and the curves (and between their reflected images of course).

here, because of the time consuming character of these calculations. However, one can expect an increase in the crystallization zone for these envelopes similar to that of Fig. 7h. 


\section{CONCLUSION AND OUTLOOK}

We have presented calculations for anions and cations near a spherical water-oil interface, taking into account ionic self-energies and screening, to describe the spontaneous charging of water droplets in oil. This theory was applied to emulsions of oil and water which contain ions.

In the extremely dilute droplet limit, the effects of curvature on the charge and excess interfacial tension induced by ion partitioning of the anions and cations were compared to results obtained for a planar interface. It turns out that the planar limit approximation used in Ref. 6] can be applied with a high degree of accuracy for many system parameters. However, in the range of the experiments of Refs. [3, 4], we have shown that spherical and planar results differ significantly. Water-inoil droplets have a substantially higher and oil-in-water droplets a substantially lower charge/excess interfacial tension than one would expect on the basis of planar calculations. In accordance with Ref. [27] we found that the value of physical quantities in a spherical system can to an extent be approximated using a polynomial expansion in $1 /\left|\kappa_{o} a\right|$ around the planar value.

For finite volume fractions of water in oil we have investigated the crystallization of water-in-oil droplets using the dimensionless coupling parameter $\Gamma$ of a pointYukawa system to predict crystallization [22]. The range in parameter space in which crystal formation can occur is greatly extended by using spherical values w.r.t. the planar result, mainly because of the larger surface charge densities in the spherical case. We expect that the theory we have presented captures the physics of the experi- ments performed by Leunissen et al. in Refs. [3, 4]. However, quantitative comparison between theory and experiment is not possible at this time. Not only additional theoretical effort is required, e.g., including more realistic self-energies [17] or a wider class thereof [14, 15, 16], but the complex chemistry in these oil-water emulsions needs to be further scrutinized and elucidated experimentally to facilitate such a comparison.

Another extension of the present theory for ions in the vicinity of a curved oil-water interface is the addition of charged colloidal particles. It was shown experimentally [3, 4] and theoretically in the planar geometry [4, 6] that the phenomenology due to the presence of charged colloids is extremely rich, e.g. involving strong colloidal adsorption at the droplet surface with adjacent huge colloid-free zones. One would expect curvature effects in these systems as well. Moreover, the much smaller oilin-water droplets in the $10-100 \mathrm{~nm}$ range as observed in the Pickering emulsions of Refs. [28, 29] certainly warrant a theoretical treatment that takes the finite curvature of the droplets into account. Studies along these lines are in progress.

\section{ACKNOWLEDGEMENTS}

It is a pleasure to thank M. E. Leunissen and A. van Blaaderen for useful discussions and sharing unpublished experimental data with us. This work is part of the research program of the 'Stichting voor Fundamenteel Onderzoek der Materie (FOM)', which is financially supported by the 'Nederlandse Organisatie voor Wetenschappelijk Onderzoek (NWO)'.
[1] S. Pickering, J. Chem. Soc. 92, 2001 (1907).

[2] Modern Aspects of Emulsion Science (The Royal Society of Chemistry: Cambridge, 1998), 1st ed., edited by B. P. Binks.

[3] M. E. Leunissen et al., Proc. Natl. Acad. Sci. U.S.A. 104, 2585 (2007).

[4] M. E. Leunissen et al., Phys. Chem. Chem. Phys. 9, 6405 (2007).

[5] M. E. Leunissen, Private communication.

[6] J. Zwanikken and R. van Roij, Phys. Rev. Lett. 99, 178301 (2007).

[7] M. Born, Z. Phys. 1, 45 (1920).

[8] E. J. W. Verwey and K. F. Niessen, Phil. Mag. J. Sci. 28, 435 (1939).

[9] J. N. Israelachvili, Intermolecular And Surface Forces (Academic Press: London, 1992), 2nd ed.

[10] A. L. Nichols III and L. R. Pratt, J. Chem. Phys. 80, 6225 (1984).

[11] B. I. Shklovskii, Phys. Rev. Lett. 82, 3268 (1999).

[12] B. I. Shklovskii, Phys. Rev. E 60, 5802 (1999).

[13] A. Y. Grosberg, T. T. Nguyen, and B. I. Shklovskii, Phys. Mod. Phys. 74, 329 (2002).

[14] A. Onuki, Phys. Rev. E 73, 021506 (2006).

[15] A. Onuki, J. Chem. Phys. 128, 224704 (2008).
[16] A. Onuki, Europhys. Lett. 82, 58002 (2008).

[17] D. Horinek and R. R. Netz, Phys. Rev. Lett. 99, 226104 (2007).

[18] W. Kung, F. J. Solis, and M. Olvera de la Cruz, arXiv:0710.0369v2 (2007), J. Zwanikken et al. (unpublished).

[19] S. Alexander et al., J. Chem. Phys. 80, 5776 (1984).

[20] R. Evans, Adv. Phys. 28, 143 (1979).

[21] R. Evans, Liquides aux interfaces / Liquids at interfaces: Les Houches, Session XLVIII 1988 (North-Holland: Amsterdam, 1989), p. 1, edited by J. Charvolin, J. F. Joanny, and J. Zinn-Justin.

[22] O. S. Vaulina and S. A. Khrapak, J. Exp. and Theor. Phys. 90, 287 (2000).

[23] P. M. Dove and C. J. Nix, Geochimica et Cosmochimica Acta 61, 3329 (1997).

[24] B. E. Conway, Ionic Hydration in Chemistry and Biophysics (Elsevier Science Ltd: New York, 1981), 1st ed.

[25] Y. Marcus, J. Solution Chem. 12, 271 (1983).

[26] M. Bier, J. Zwanikken, and R. van Roij, Phys. Rev. Lett. 101, 046104 (2008).

[27] P.-M. König, R. Roth, and K. R. Mecke, Phys. Rev. Lett. 93, 160601 (2004).

[28] S. Sacanna, W. K. Kegel, and A. P. Philipse, Phys. Rev. 
Lett. 98, 158301 (2007).

[29] S. Sacanna, W. K. Kegel, and A. P. Philipse, Langmuir

23, 10486 (2007). 